\title{
Rib 12
}

National Cancer Institute

\section{Source}

National Cancer Institute. Rib 12. NCI Thesaurus. Code C52767.

The twelfth rib counting from the top of the rib cage down. 